\title{
Reconstrucción de precipitación y temperatura con anillos de crecimiento anual del ciprés Taxodium mucronatum (Taxodiaceae) en Coahuila, México
}

\author{
José Villanueva-Díaz ${ }^{1 *}$, Aldo Rafael Martínez-Sifuentes ${ }^{1}$, Fátima del Rocío Reyes-Camarillo ${ }^{1}$ \\ \& Juan Estrada-Ávalos ${ }^{1}$ \\ 1. Instituto Nacional de Investigaciones Forestales, Agrícolas y Pecuarias, Centro Nacional de Investigación Disciplinaria \\ Relación Agua-Suelo-Planta-Atmósfera (INIFAP-CENID-RASPA), Margen Derecha del Canal Sacramento Km 6.5, \\ Gómez Palacio, Durango, México; villanueva.jose@inifap.gob.mx (*Correspondencia), im_aldo09@hotmail.com, \\ fatimareyesca@gmail.com, estrada.juan@inifap.gob.mx
}

Recibido 28-VII-2020. Corregido 03-XII-2020. Aceptado 11-XII-2020.

\begin{abstract}
Reconstruction of precipitation and temperature with annual growth rings of the cypress Taxodium mucronatum (Taxodiaceae) in Coahuila, Mexico. Introduction: Dendroclimatic reconstructions in water-yield basins lacking hydroclimatic data, such as the Rio Sabinas is important to analyze its interannual and multiannual climatic variability. One of the species useful for this purpose is the Montezuma baldcypress (Taxodium mucronatum Ten.), a long-lived species with well-defined annual rings, present along the riparian zone of the Rio Sabinas that constitutes a "proxy" of interannual and multiannual climate variability. Objective: develop a seasonal precipitation and maximum temperature reconstructions, and to analyze the influence of global circulatory modes on the species annual radial increase. Methods: Increment cores of the Montezuma baldcypress specimens were dated through dendrochronological techniques to produce a ring-width series. Climate gridded data from two databases were used to develop a response function analysis for climate reconstruction purposes. Results: A ring-width chronology extending from 1808 to 2018 (211 years) was developed and used to develop a seasonal April-June precipitation and a mean June-July maximum temperature reconstruction. The driest period detected on the rainfall reconstruction occurred from 1815 to 1818 with $27.4 \mathrm{~mm}$ and a maximum temperature of $41.2^{\circ} \mathrm{C}$; while the wettest year was 1828 with $393.72 \mathrm{~mm}$. The ring-width chronology was correlated with the Standardized Precipitation Evaporation Index (SPEI) and the Palmer Drought Severity Index (PDSI), and with indices of atmospheric phenomena such as El Niño Southern Oscillation (ENSO), through the Southern Oscillation Index (SOI) and the Multivariate Index (MEI); Pacific Decadal Oscillation (PDO), and the Atlantic Multidecadal Oscillation (AMO). The relationship between the ring-width series and drought indices (SPEI, PDSI) was significant in June $(\mathrm{r}=0.52, \mathrm{P}<0.01)$, and June-August $(\mathrm{r}=0.38, \mathrm{P}<0.05)$ for the SPEI and reconstructed PDSI, respectively. It was found a significant association between the ring-width chronology and the reconstructed November-February SOI $(r=-0.41, \mathrm{P}<0.01)$. The Pacific Decadal Oscillation and the Atlantic Multidecadal Oscillation were not significant. Conclusions: On this study, we developed a climatic response function and reconstructed seasonal climatic variables (precipitation, maximum temperature) of importance to develop management strategies for conservation of the Montezuma bald cypress on this basin, and to implement mitigation actions for the presence of extreme climatic events that may occur in coming years.
\end{abstract}

Key words: Mexican baldcypress; tree-rings; climatic reconstruction; extreme hydroclimatic events.

Villanueva-Díaz, J., Martínez-Sifuentes, A.R., Reyes-Camarillo, F.R., \& Estrada-Ávalos, J. (2021). Reconstrucción de precipitación y temperatura con anillos de crecimiento anual del ciprés Taxodium mucronatum (Taxodiaceae) en Coahuila, México. Revista de Biología Tropical, 69(1), 302-316. DOI 10.15517/rbt.v69i1.43249 
El análisis del clima en cuencas productoras de agua mediante estudios dendrocronológicos es relevante para determinar su comportamiento en el tiempo, analizar tendencias con fines predictivos y la influencia que ejercen fenómenos de circulación global en su comportamiento (Anderson, Ogle, Tootle, \& Oubeidillah, 2019). Esto permite generar información más allá de los registros climáticos, que son generalmente cortos (menos de 70 años) y que no alcanzan a captar la variabilidad presente en series climáticas más extensas, como las generadas con anillos de crecimiento, que se pueden extender por siglos y que son de utilidad para determinar la frecuencia con que se presentan eventos hidroclimáticos extremos y los cambios en el comportamiento del clima debido al calentamiento global.

La región del Río Sabinas en Coahuila, en el que se distribuye una especie ribereña conocida como ciprés, sabino o ahuehuete (Taxodium mucronatum Ten.), la cual ha sido previamente utilizada para estudios dendroclimáticos e hidrológicos, constituye una opción para determinar la variación climática por cientos de años e inclusive milenios, ya que es la especie más longeva en México (Correa-Díaz et al., 2014; Osorio-Osorio, Astudillo-Sánchez, Villanueva-Díaz, Soria-Díaz, \& Vargas-Tristan, 2020; Villanueva-Díaz et al., 2020) y una de las especies milenarias en el Este de los Estados Unidos, donde alcanza más de 2600 años de edad (Stahle et al., 2019).

En términos dendrocronológicos, la sensibilidad climática de la especies no es tan alta como la que poseen diversas especies de coníferas de climas semiáridos (VillanuevaDíaz et al., 2007), pero esto no es un factor limitante para reconstrucciones hidroclimáticas (Fritts, 1976), ya que la especie se ha utilizado para desarrollar reconstrucciones centenarias de precipitación en regiones del centro y norte de México y definido la influencia que ejercen fenómenos de circulación global, donde uno con mayor impacto en el norte de México es El Niño Oscilación del Sur (ENSO, por sus siglas en inglés), el cual determina la variabilidad hidroclimática en los rangos interanuales y multianuales (Magaña, Pérez, Vázquez, Carrizosa, \& Pérez, 1999; Stahle et al., 2016). La tele-conexión de ENSO en su fase cálida (El Niño) y la fase fría (La Niña), tienen gran influencia en la precipitación de inviernoprimavera y de verano en el norte de México (Méndez \& Magaña, 2010; Stahle et al., 2011) y es relevante para la producción de biomasa de especies forestales, cuyo incremento anual depende de la lluvia en dicho período (Castruita et al., 2019).

El Río Sabinas en el norte del estado de Coahuila constituye la principal fuente de agua del Distrito de Riego 004 (DDR 004), con el que se irriga una superficie cercana a 30000 ha en los estados de Coahuila y Nuevo León (Comisión Nacional del Agua, 2013); por lo que es importante un entendimiento de la variabilidad climática de alta y baja frecuencia, su tendencia en el tiempo y el potencial impacto del calentamiento global (Seager et al., 2009).

Cambios drásticos en el uso del suelo ocasionados por la explotación de carbón mineral, pastoreo intensivo y contaminación de sus tributarios han originado la fragmentación de las áreas riparias, donde se ubican los bosques de galería con T. mucronatum, provocando la mortandad de esta especie en transectos del Río Sabinas (Villanueva-Díaz, Constante, Cerano, \& Martínez, 2014). T. mucronatum y especias asociadas aporta grandes beneficios a los bosques de galería, al fomentar la biodiversidad, mantener el cauce estable, crear un microclima especial, favorecer el filtrado de contaminantes, captura de carbono, entre otros beneficios adicionales (Granados-Sánchez, HernándezGarcía, \& López-Ríos, 2006).

Dada la longevidad de T. mucronatum y la limitada disponibilidad de registros climáticos; el presente estudio tiene como objetivo analizar la variabilidad estacional de la precipitación y temperatura que afecta la cuenca del Río Sabinas en los últimos siglos y determinar la variación interanual y multianual, e influencia en dicha variabilidad de fenómenos circulatorios océano-atmósfera. La hipótesis de este estudio se fundamenta en que los anillos de crecimiento de T. mucronatum constituyen un "proxy" 
de la precipitación y temperatura en el área de provisión hídrica de la cuenca del Río Sabinas y que su reconstrucción, permitirá analizar la presencia de eventos climáticos extremos y sus tendencias con fines de planeación de los recursos hídricos en esta cuenca.

\section{MATERIALES Y MÉTODOS}

Área de estudio: El área de estudio se localiza en la región carbonífera de los municipios de Sabinas y San Juan de Sabinas en el Estado de Coahuila, México, dentro de la Región Hidrológica 24 Bravo-Conchos entre las coordenadas extremas $\left(27^{\circ} 09^{\prime} 18^{\prime \prime}\right.$ $28^{\circ} 57^{\prime} 58^{\prime \prime} \mathrm{N} \& 100^{\circ} 35^{\prime} 33^{\prime}$ " - 102 23 '53” W) (Fig. 1). De acuerdo con Köppen modificado por García (2004), el clima es semiárido y semicálido de tipo BS1hw, con una precipitación media anual de 400 a $600 \mathrm{~mm}$ y una temperatura media anual de 20 a $22{ }^{\circ} \mathrm{C}$, con una elevación de 410 msnm (INEGI, 1983). La vegetación está constituida por pastizal natural, bosque de galería, con presencia de Taxodium mucronatum Ten., Platanus mexicana, var. Interior Nixon \& J.M. Poole, Carya illinoinensis (Wangenh) K.K, Salix goodingii C.R. Ball y Cephalantus occidentalis L (Villareal, Carranza, Estrada, \& Rodríguez, 2006).

\section{Obtención de núcleos de crecimiento:}

Los sitios de muestreo fueron tres parajes ubicados en los márgenes del Río Sabinas dominados por bosques de galería de T. mucronatum. Los sitios fueron Santa María, Las Adjuntas y en el margen propio del Río Sabinas (Fig. 1). En los sitios de muestreo, se seleccionaron 70 individuos de T. mucronatum con características de longevidad y sin evidencia de disturbio, a los cuales se les extrajo de 2 a 3 núcleos de crecimiento por individuo. Los núcleos de crecimiento se obtuvieron con un taladro de Pressler, marca Haglöf a la altura del pecho $(1.30 \mathrm{~m})$. El datado y medición de
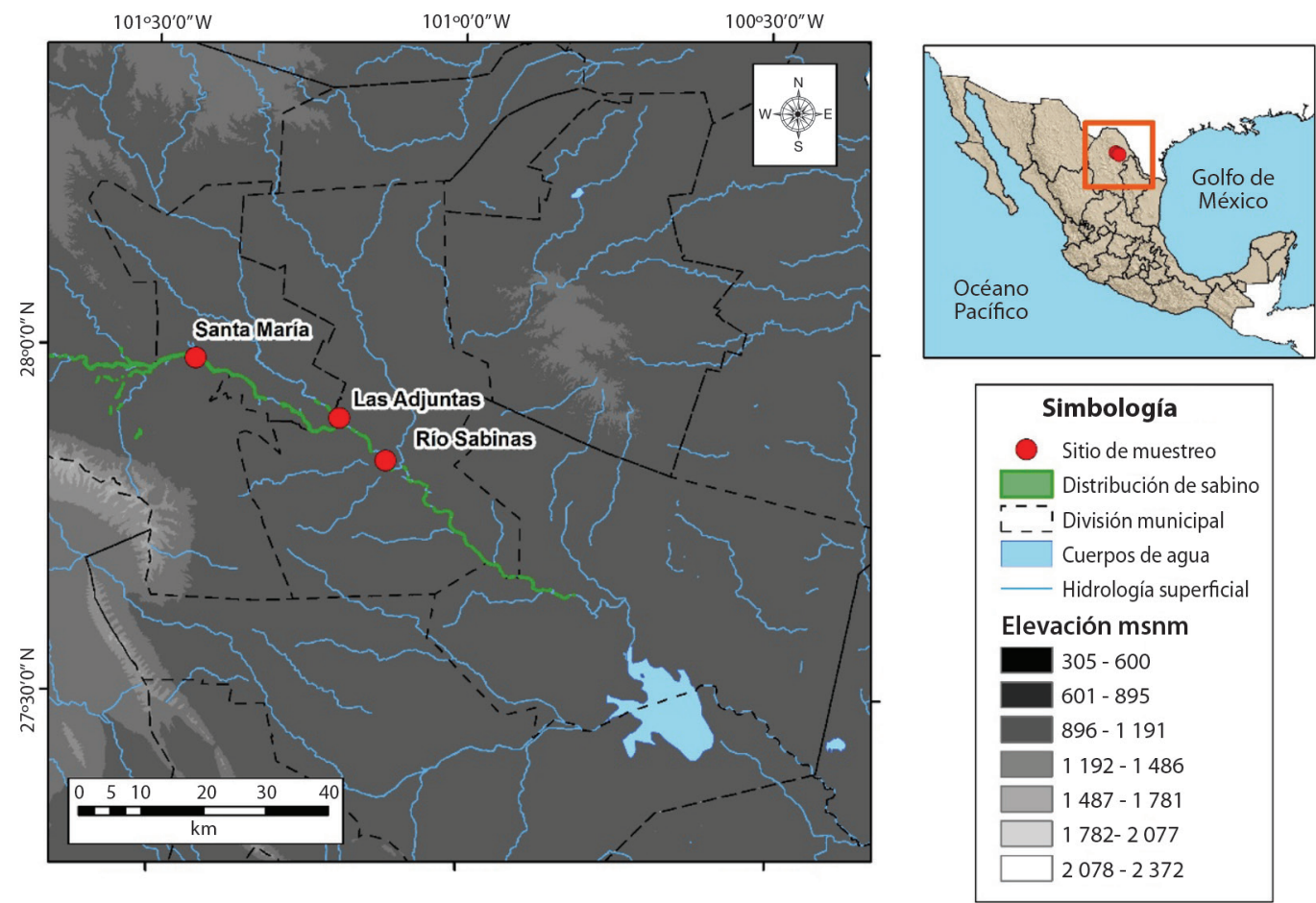

Fig. 1. Ubicación geográfica de zona de estudio en la región carbonífera del estado de Coahuila.

Fig. 1. Geographic location of the study zone in the region carbonifera of the Coahuila state. 
las muestras extraídas, se realizó mediante técnicas dendrocronológicas convencionales (Stokes \& Smiley, 1968). La medición de los anillos de crecimiento, se realizó con un sistema de medición marca Velmex Inc. TA con una precisión de $0.001 \mathrm{~mm}$; la calidad del fechado se verificó a través del software original de COFECHA escrito en 1982 (Holmes, 1983) y actualizado a windows XP en 2013, y la estandarización de las series de crecimiento con el software ARSTAN (Cook, 1987) en su versión actualizada 2014 para Windows XP. Se determinaron estadísticos dendrocronológicos como intercorrelación entre series (medición de la señal climática común de las series), sensibilidad media (diferencia relativa en el grosor de un anillo de crecimiento con relación al siguiente), deviación estándar (medida de dispersión del valor de ancho de anillo con relación a un valor medio), autocorrelación de primer orden (relación entre el crecimiento del anillo actual y el del año previo) y Señal Expresada de la población (EPS, por sus siglas en inglés), indicador de correspondencia entre la varianza de la cronología con la población teórica, el cual deberá ser EPS $\geq 0.85$ (Mérian, Pierrat, \& Lebourgeois, 2013); y Rbar, que estima el porcentaje de la varianza común entre las series; los estadísticos fueron obtenidos usando la librería dplR del software R versión 1.7.1 ( $\mathrm{R}$ Core Team, 2014; Bunn et al., 2019).

Información climática: La información de precipitación se obtuvo del grid desarrollado por Terán-Cuevas (2010), específicamente de los centroides (47206), (47208) y (46889), estos se promediaron con la finalidad de obtener una precipitación media mensual regional, propuesta como representativa del área de estudio, con registros continuos de 1950 a 2015. La información de temperatura se obtuvo de la base de datos de alta resolución del ClimateNA versión 5.21, que incluye temperatura máxima, mínima y media para el período 1901 a 2013 (Wang, Hamann, Spittlehouse, \& Carroll, 2016).

La asociación entre la cronología desarrollada de $T$. mucronatum y la información climática obtenida, se realizó a través del programa Dendroclim2002 (Biondi \& Waikul, 2004) para un período de 19 meses consecutivos, es decir, siete meses previos al año de crecimiento y 12 meses durante el año de crecimiento. Con base a la asociación significativa $(\mathrm{P}<0.01)$ entre la cronología y variables climáticas, se generó un modelo de transferencia, el cual se validó mediante el proceso de calibración-verificación con la subrutina "verify" de la Librería de Programas Dendrocronológicos de la Universidad de Arizona (Holmes, 1983).

La presencia de picos o frecuencias significativas en la cronología, se determinó mediante un análisis espectral de potencia con el software R, versión 3-4.3, con la finalidad de identificar ciclicidades en eventos de baja frecuencia a lo largo de la serie dendrocronológica, la cual permite analizar la influencia en el crecimiento de fenómenos atmosféricos de circulación global (Grinsted, Moore, \& Jevrejeva, 2004). Para determinar los períodos en los que la reconstrucción evidencia frecuencias significativas, se desarrolló un Análisis Espectral de Ondeleta con el software R, versión 0.20.15 (Tarik, Aslak, \& Viliam, 2017).

La asociación entre la serie dendrocronológica y condiciones de sequía, se determinó través del Índice Estandarizado de Precipitación-Evapotranspiración (SPEI, por sus siglas en inglés), el cual es un índice sensitivo a las alteraciones históricas de precipitación y temperatura (Vicente-Serrano, Beguería, LópezMoreno, Angulo, \& Kenawy, 2010). El índice fue descargado del monitor global de sequías a una escala temporal de tres meses, lo cual favorece un análisis más real del balance de humedad en el suelo (Serrano-Barrios, VicenteSerrano, Flores-Magdaleno, Tijerina-Chávez, \& Vázquez-Soto, 2016).

La cronología fue comparada con el Índice de Severidad de Sequías de Palmer reconstruido (PDSI, por sus siglas en inglés), el cual constituye un balance hidrológico que considera parámetros como precipitación, escurrimiento, humedad y evaporación. Este índice se obtuvo del Atlas Mexicano de Sequía (Stahle et al., 2016). La cronología también se contrastó 
con el Índice de Severidad de Sequía de Palmer Auto-Calibrado (sc-PDSI, por sus siglas en inglés), el cual contempla todas las variables y características del PDSI de Palmer, con la diferencia es que se desarrolla a nivel mensual, con base a cambios en el régimen climático de cada estación utilizada (Van der Schrier, Barichivich, Briffa, \& Jones, 2013), complementada con datos actualizados al 2018 del Climatic Research Unit (Harris, Jones, Osborn, \& Lister, 2014). La comparación se efectuó mediante correlación espacial (Wells, Goddard, \& Hayes, 2004).

La asociación entre índices de ENSO y la cronología, se realizó con el Índice de Oscilación del Sur Reconstruido (SOI, por sus siglas en inglés) (Ropelewski \& Jones, 1987) y el Índice Multivariado (MEI, por sus siglas en inglés) (Wolter \& Timlin, 2011). Adicionalmente, se realizó una comparación de la cronología con índices de la Oscilación Decadal del Pacífico (PDO, por sus siglas en inglés), el cual se presenta como una función ortogonal empírica de las anomalías mensuales de la temperatura de la superficie del Océano Pacífico a partir del paralelo a $20^{\circ}$ hasta el Polo Norte (Mantua, Hare, Zhang, Wallace, \& Francis, 1997). Otro de los fenómenos considerados fue la Oscilación Multidecadal del Atlántico
(AMO, por sus siglas en inglés), que involucra las anomalías promedio de la temperatura de la superficie en la cuenca del Atlántico norte $\left(0-10^{\circ} \mathrm{N}\right)$ en ciclos estimados de 60 a 80 años (Trenberth \& Zhang, 2019).

\section{RESULTADOS}

Los parámetros dendrocronológicos de la serie de anillo total mostraron potencial para desarrollar una reconstrucción hidroclimática de más de dos siglos de extensión (Tabla 1). La cronología se extendió de 1808 a 2018 (211 años), a la cual se le ajustó una curva decenal flexible "Spline" para denotar eventos de baja frecuencia (Fig. 2).

Función de respuesta climática: El análisis de función de respuesta llevado a cabo con Dendroclim2002, presentó asociaciones significativas con la precipitación mensual de los meses de agosto $(\mathrm{r}=0.38, \mathrm{P}<0.05)$, septiembre $(\mathrm{r}=0.45, \mathrm{P}<0.05)$ y noviembre $(\mathrm{r}=0.28$, $\mathrm{P}<0.05)$ del año previo, y marzo $(\mathrm{r}=0.27$, $\mathrm{P}$ $<0.05)$, junio $(\mathrm{r}=0.46, \mathrm{P}<0.05)$ y julio $(\mathrm{r}=$ $0.25, \mathrm{P}<0.05)$, del año actual de crecimiento. Estacionalmente, la mayor asociación se obtuvo con la precipitación acumulada abril-junio ( $\mathrm{r}$ $=0.71, \mathrm{P}<0.05)($ Fig. 3).

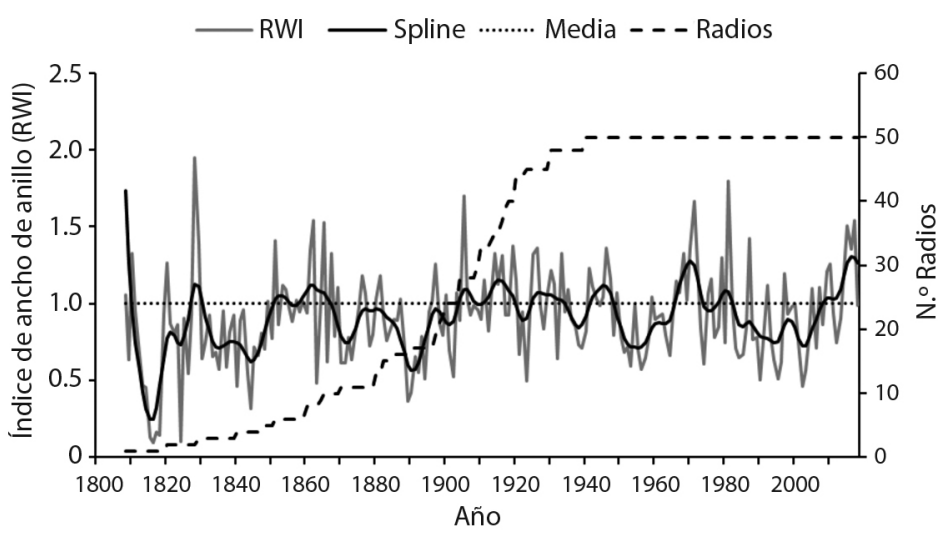

Fig. 2. Cronología de anillo total versión estándar de especímenes de Taxodium mucronatum (Ahuehuete) muestreados en parajes del Río Sabinas, Coahuila.

Fig. 2. Standard ring-width chronology of the Mexican baldcypress (Taxodium mucronatum) specimens sampled in the Sabinas River, Sabinas, Coahuila. 
TABLA 1

Parámetros dendrocronológicos de los anillos de crecimiento de Taxodium mucronatum (Ahuehuete) de especímenes recolectados en diversos parajes del Río Sabinas, Coahuila

TABLE 1

Dendrochronological parameters of the Mexican baldcypress (Taxodium mucronatum) tree-rings specimens collected in several stands of the Rio Sabinas, Coahuila

\begin{tabular}{ccccccc} 
Sitio & $\begin{array}{c}\text { Intercorrelación } \\
\text { entre series }\end{array}$ & $\begin{array}{c}\text { Sensibilidad } \\
\text { media }\end{array}$ & $\begin{array}{c}\text { Desviación } \\
\text { estándar }\end{array}$ & $\begin{array}{c}\text { Autocorrelación } \\
\text { de primer orden }\end{array}$ & Rbar & EPS \\
Río Sabinas & 0.468 & 0.457 & 0.467 & 0.656 & 0.263 & 0.877 \\
\hline
\end{tabular}
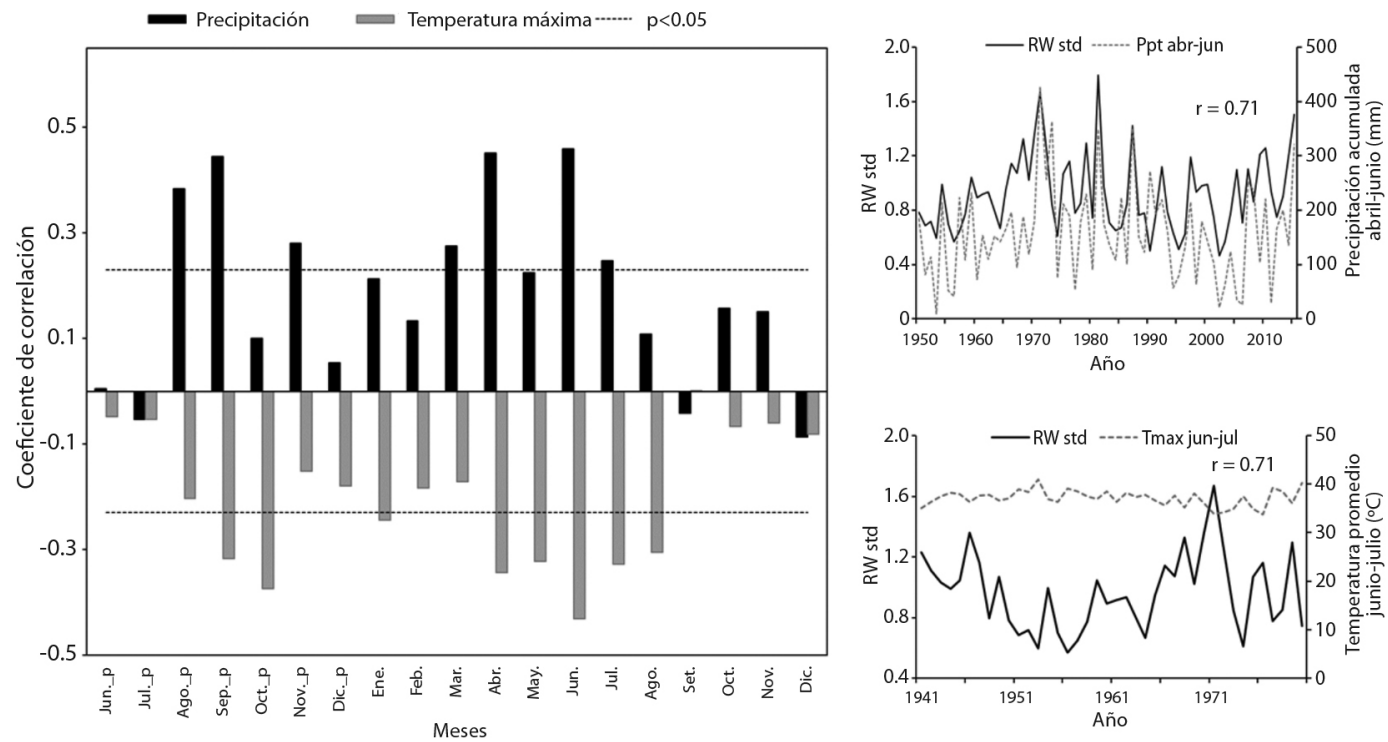

Fig. 3. Asociación climática entre la cronología y las variables precipitación y temperatura máxima. Los valores que se ubican por encima y por debajo de la línea horizontal punteada son significativos $(\mathrm{P}<0.05)$.

Fig. 3. Climatic association between the ring-width chronology, precipitation and maximum temperature. Values above and below of the horizontal dotted lines are significant $(\mathrm{P}<0.05)$.

La asociación entre la cronología desarrollada y temperatura máxima mensual fueron negativas, pero significativa durante agosto $(\mathrm{r}$ $=-0.23 . \mathrm{P}<0.05)$, septiembre $(\mathrm{r}=-0.32, \mathrm{P}<$ $0.05)$ y octubre $(\mathrm{r}=-0.37, \mathrm{P}<0.05)$ del año previo, y durante el año actual de crecimiento en los meses de enero $(\mathrm{r}=-0.24, \mathrm{P}<0.05)$, abril $(\mathrm{r}=-0.34, \mathrm{P}<0.05)$, mayo $(\mathrm{r}=-0.32, \mathrm{P}<0.05)$, junio $(\mathrm{r}=-0.43, \mathrm{P}<0.05)$, julio $(\mathrm{r}=-0.33$, $\mathrm{P}<0.05)$ y agosto $(\mathrm{r}=-0.31, \mathrm{P}<0.05)$; $\sin$ embargo, el periodo con mayor correlación se encontró con la temperatura máxima de verano junio-julio ( $\mathrm{r}=-0.71, \mathrm{P}<0.05)$ (Fig. 3). Por lo que respecta a la temperatura mínima y media, no se detectó significancia $(\mathrm{P}>0.05)$.

Modelos de reconstrucción climática: Para la reconstrucción de precipitación, se consideró el periodo acumulado abril-junio, que representa $51.06 \%$ del total de la varianza del modelo. Con relación a temperatura máxima, se consideró el periodo junio-julio, cuyo modelo explica $50.14 \%$ de la varianza; las ecuaciones de transferencia son las siguientes (Fig. 4): 


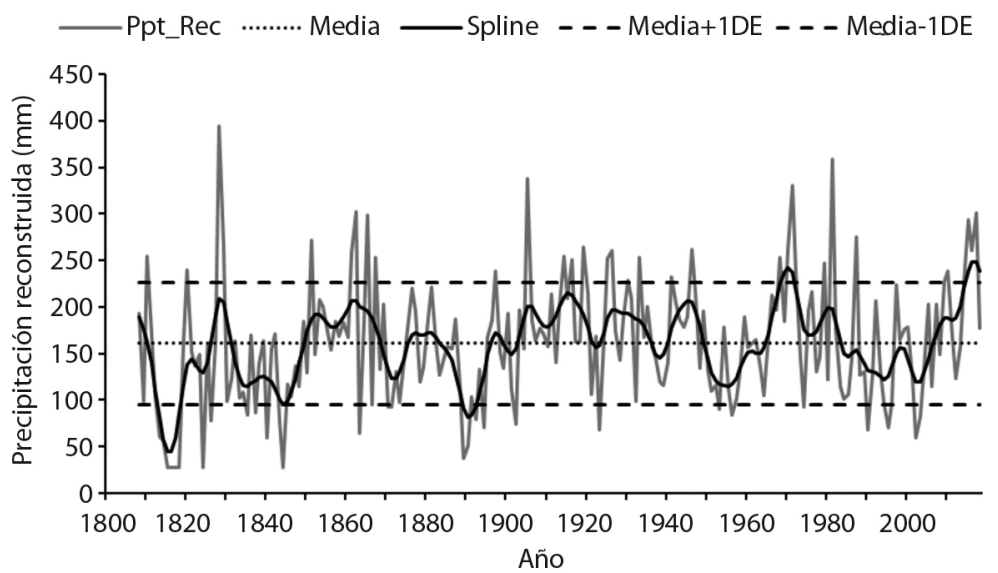

Fig. 4. Precipitación estacional abril-junio reconstruida con registros climáticos de la cuenca del Río sabinas, Coahuila. La línea horizontal punteada es la media de la reconstrucción y las líneas paralelas superior e inferior representan una desviación estándar por encima y por debajo de la media para definir eventos extremos (muy húmedos o muy secos). La línea flexible es una curva decenal ajustada a los valores estacionales para resaltar eventos decenales de baja frecuencia.

Fig. 4. Seasonal April-June reconstructed precipitation with climatic records of the Sabinas River, Coahuila. The horizontal dotted line is the reconstruction mean and the upper and lower parallel lines are one standard deviation above- and below the reconstructed mean to define extreme events (very wet or very dry). The flexible line is a 10-year spline to highlight low frequency events.

\section{Precipitación estacional abril-junio}

$\mathrm{Yi}=-44.324+224.41 * \mathrm{Xi}$; donde, $\mathrm{Yi}=$ precipitación estacional reconstruida abril-junio $(\mathrm{mm})$ y $\mathrm{Xi}=$ índice de anillo total para un año específico.

\section{Temperatura máxima estacional junio-julio}

$\mathrm{Yi}=41.6066-4.6323^{*} \mathrm{Xi}$; donde $\mathrm{Yi}=$ temperatura máxima reconstruida del período estacional junio-julio $\left({ }^{\circ} \mathrm{C}\right)$ y $\mathrm{Xi}=$ índice de anillo total para un año en específico.

Los estadísticos de validación de ambos modelos resultaron significativos (Tabla 2), por lo que se usaron con fines de reconstrucción de precipitación y temperatura máxima estacional, respectivamente.

Reconstrucción estacional de precipitación abril-junio: La reconstrucción estacional

TABLA 2

Estadísticos para el proceso de validación de los modelos de reconstrucción de precipitación y de temperatura estacional

TABLE 2

Statistics for the validation process of the precipitation and seasonal temperature reconstruction models

\begin{tabular}{|c|c|c|c|c|c|c|}
\hline Periodo & $\begin{array}{l}\text { Coeficiente de } \\
\text { correlación de Pearson }\end{array}$ & $\begin{array}{l}\text { Reducción del } \\
\text { error }\end{array}$ & Valor de " $\mathrm{t}$ " & $\begin{array}{l}\text { Prueba de } \\
\text { signos }\end{array}$ & $\begin{array}{c}\text { Primera diferencia } \\
\text { significativa }\end{array}$ & $\begin{array}{l}\text { Coeficiente de } \\
\text { Durbin-Watson }\end{array}$ \\
\hline \multicolumn{7}{|c|}{ Precipitación } \\
\hline 1950-1969 & $0.50 *$ & $0.14^{*}$ & $1.95^{*}$ & $7 \mathrm{NS}$ & $3 *$ & 0.69 \\
\hline 1970-1989 & $0.75^{*}$ & $0.67 *$ & $2.38^{*}$ & $4 *$ & $4 *$ & 1.69 \\
\hline \multicolumn{7}{|c|}{ Temperatura máxima } \\
\hline $1941-1960$ & $0.68 *$ & $0.40^{*}$ & $2.88^{*}$ & $7 \mathrm{NS}$ & $1 *$ & 0.94 \\
\hline $1961-1980$ & $0.69 *$ & $0.59 *$ & $3.16^{*}$ & $6 \mathrm{NS}$ & $2 *$ & 0.78 \\
\hline
\end{tabular}

*Significativo $(\mathrm{P}<0.05)$; NS: no significativo. 
generada, muestra alta variabilidad interanual y multianual del periodo primavera e inicios de verano para la región del Río Sabinas (Fig. 4). La media histórica reconstruida fue de 160.82 $\mathrm{mm}$, lo que permitió identificar años extremos de sequía (valores una desviación estándar por debajo de la media) y húmedos (una desviación estándar por encima de la media). Entre los periodos considerados como secos, se determinaron los siguientes: 1813-1818, 1824, 1826, $1835,1837,1840,1843-1844,1863,1870-$ 1871, 1889-1890, 1892, 1894, 1902, 1923, 1953, 1956, 1974, 1990, 1995, 2002-2003, donde el periodo de 1815-1818 fue el catalogado como más seco con una precipitación media de $27.4 \mathrm{~mm}$ en el período abril-junio; seguido por el año de 1889 con $36.92 \mathrm{~mm}$ y 2002-2003 con un promedio de $71.34 \mathrm{~mm}$. Eventos húmedos extremos ocurrieron en 1810, 1820, 1828$1829,1851,1861-1862,1865,1867,1897$, 1905, 1914, 1919, 1925-1926, 1930, 1933, 1946, 1968, 1970-1972, 1979, 1981, 1987, 2009-2010, 2015-2017, donde el año de 1828 fue el más húmedo con $393.72 \mathrm{~mm}$, seguido de 1981 con $358.73 \mathrm{~mm}$ y $2015-2017$ con una precipitación media de $285.19 \mathrm{~mm}$.
Reconstrucción estacional de temperatura máxima junio-julio: La reconstrucción de temperatura máxima de verano en la cuenca hidrológica del Río Sabinas, mostró fluctuaciones en un rango de 32.56 a $41.16{ }^{\circ} \mathrm{C}$, con una media histórica reconstruida de $37.39{ }^{\circ} \mathrm{C}$ (Fig. 5). Años extremos con temperaturas máximas ocurrieron en $1816\left(41.16^{\circ} \mathrm{C}\right)$ y $1824(41.12$ $\left.{ }^{\circ} \mathrm{C}\right)$; mientras que la temperatura máxima inferior, se presentó en $1828\left(32.56^{\circ} \mathrm{C}\right)$.

Análisis de densidad espectral y de ondeleta: Del análisis espectral de potencia, se identificaron dos picos significativos, el primero, con una frecuencia de 2.7 años, y el segundo con 45.9 años (Fig. 6A). Acorde al análisis de ondeleta generado de la serie de crecimiento de anillo total, se determinaron frecuencias significativas $(\mathrm{P}<0.05)$ (zonas delimitadas por la línea negra dentro del cono de influencia), de 2.7 años del periodo reconstruido (1825-1830), (1860-1875) y (1975-1990), así como frecuencias de 45.9 años de 1890 a 1950 (Fig. 6B).

Asociación entre serie dendrocronológica e índices sequía e índices de fenómenos océano-atmósfera: La asociación entre

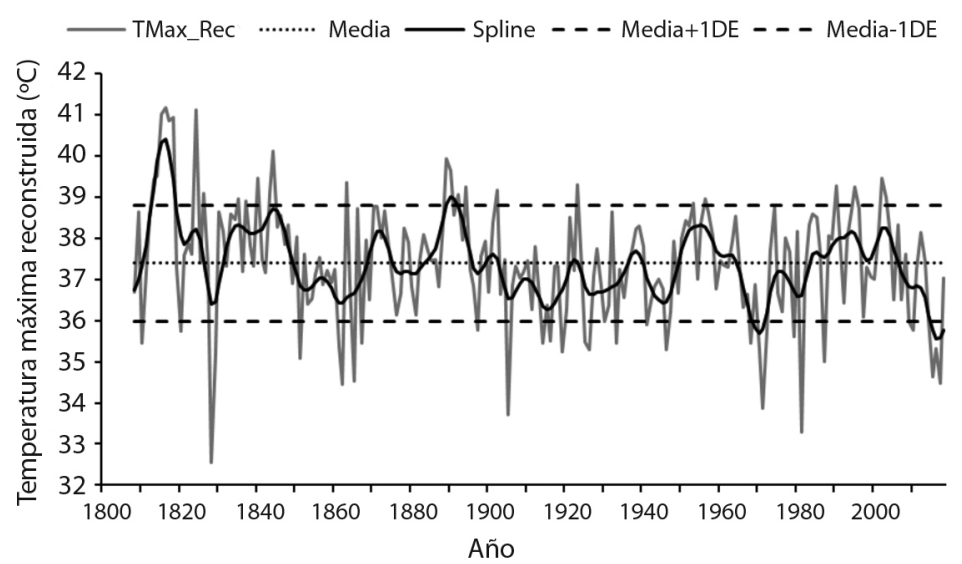

Fig. 5. Reconstrucción estacional de temperatura máxima promedio junio-julio para la zona del Río Sabinas. La línea horizontal punteada representa la media reconstruida y las líneas punteadas horizontales paralelas a la media constituyen una desviación estándar por encima y por debajo de la media, respectivamente. Valores fuera de estos límites se consideran temperaturas extremas altas y bajas, respectivamente.

Fig. 5. Seasonal reconstruction of average maximum seasonal June-July temperature for the Sabinas River. The dotted horizontal line represents the reconstructed mean, and the broken horizontal lines represents a standard deviation above and below the mean, respectively. Values outside of these limits are considered as extreme high and low events, respectively. 

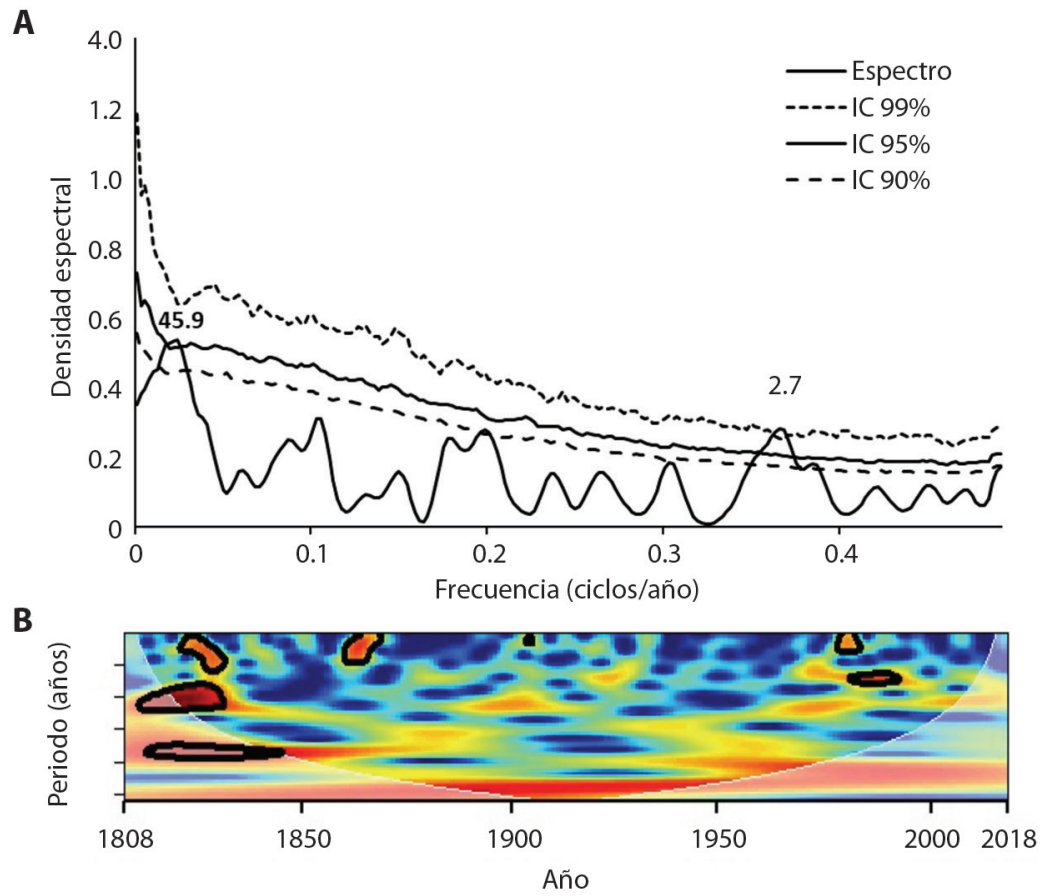

Fig. 6. A. Análisis espectral de potencia. La línea ondulada continua representa el espectro, al considerar tres niveles de significancia (90\% línea quebrada, $95 \%$ línea obscura continua y $99 \%$ línea punteada, parte superior; B. Análisis de coherencia de ondeleta, las zonas delimitadas con líneas de contorno en obscuro, indican significancia al $95 \%$.

Fig. 6. A. Power Spectral Analysis. The continuous flexible line represents the spectrum, three significant levels are considered, broken continuous line (90\%); dark continuous line (95\%), and upper dotted line (99\%); B. Wavelet Coherence Analysis, spots delimited with dark lines are significant (95\%).

la cronología de anillo total y el índice SPEI fue positiva para el mes de junio $(r=0.52$, $\mathrm{P}<0.01,1951-2018, \mathrm{~N}=68$ ). Este índice señala un patrón de influencia significativa $(\mathrm{P}<0.05)$ en sequías ocurridas en el noreste de México (Fig. 7).

La relación entre la serie dendrocronológica y el índice reconstruido PDSI del Atlas de Sequía para México, fue positivo, pero no significativo $(\mathrm{r}=0.20, \mathrm{P}>0.05,1962-2011, \mathrm{~N}$ $=50)$. Alternativamente, la correlación entre el índice calibrado del PDSI a nivel anual fue significativo $(\mathrm{r}=0.38, \mathrm{P}<0.05,1901-2018, \mathrm{~N}$ $=118$ ), mostrando un patrón similar al encontrado con el SPEI.

Asociación con fenómenos circulatorios océano-atmósfera: La relación entre la cronología de Río Sabinas y el fenómeno ENSO a través del índice reconstruido SOI noviembre-febrero fue significativo $(\mathrm{r}=-0.41, \mathrm{P}<$ 0.01, 1891-1970, $\mathrm{N}=81$ ); esta asociación, $\sin$ embargo, no fue significativa con el índice MEI $(\mathrm{r}=0.14, \mathrm{P}>0.05,1950-2017, \mathrm{~N}=68)$. La asociación del índice de anillo total y PDO no fue significativa $(\mathrm{r}=0.25, \mathrm{P}>0.05,1976-2015, \mathrm{~N}$ =40), así como con índices de AMO.

\section{DISCUSIÓN}

T. mucronatum es una especie con potencial dendrocronológico y de utilidad para desarrollar reconstrucciones de precipitación y de caudales (Stahle et al., 2012; Villanueva-Díaz et al., 2020). Esto es posible al considerar que el agua que circula por los sitios donde habita la especie, constituye el acumulado de los escurrimientos producidos por las lluvias 


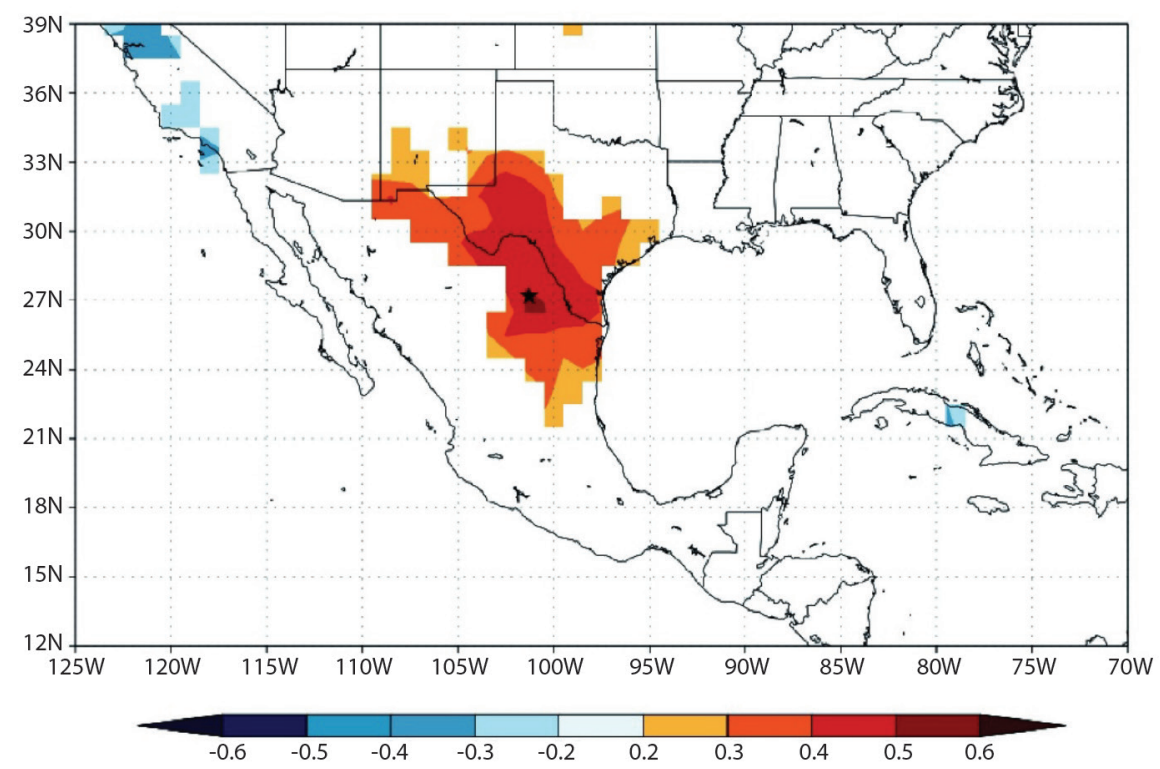

Fig. 7. Correlación espacial entre la serie de anillo total de T. mucronatum (Ahuehuete) en el Río Sabinas y el índice SPEI de junio, período de 1951 a 2018. La estrella en color negro representa el sitio de muestreo.

Fig. 7. Spatial correlation between the Mexican baldcypress (T. mucronatum) ring-width chronology from the El Rio Sabinas, and June SPEI, for the 1951 to 2018 period. The black star represents the studied site.

en las cuencas productoras de agua y son más representativos de la variación hidroclimática que caracteriza a una región hidrológica (Woodhouse \& Lukas, 2006).

T. mucronatum se distribuye de manera amplia en el territorio mexicano (Martínez, 1963), pero estudios dendrocronológicos se circunscriben en su mayoría al centro y sur de México, por lo que este estudio permite ampliar la respuesta climática de la especie en sitios poco estudiados, donde la información climática e hidrométrica es muy limitada y requiere información más extensa para determinar cambios en su variabilidad.

La cronología de anillo total generada se extendió por 211 años (1808-2018) con una intercorrelación entre series de 0.468 , sensibilidad media de 0.457 y autocorrelación de primer orden de 0.656 , valores similares a los encontrados en estudios previos con esta especie para poblaciones del centro y norte de México (Stahle et al., 2011; Correa-Díaz et al., 2014; Villanueva-Díaz et al., 2020). Estos parámetros tienen que ver con el hábitat ripario de la especie y formación de contrafuertes en el fuste principal, que generan irregularidad en el crecimiento, lo que reduce la intercorrelación y sensibilidad media dentro de radios de un mismo árbol, así como entre árboles.

Los problemas de crecimiento, sin embargo, no constituyeron una limitante para que la especie respondiera a la precipitación estacional abril-junio del año actual de crecimiento, período en el que se presenta el $34 \%$ de la precipitación anual (Terán-Cuevas, 2010). Por otra parte, la temperatura máxima promedio estacional junio-julio mostró un efecto negativo en el crecimiento de la especie; de esta manera, menor temperatura y mayor precipitación interactúan para generar mayores incrementos radiales y viceversa. La precipitación de primavera resulta de gran relevancia para el crecimiento de la especie, la cual, al ser de baja intensidad y quedar almacenada en el perfil del suelo, resulta fácilmente aprovechable para la especie, que en esa época se encuentra en plena etapa de incremento radial (VillanuevaDíaz, Constante, Cerano, Estrada, \& Tostado, 
2013). Por el contrario, máximas temperaturas en junio y julio incrementan la evapotranspiración, provocan estrés hídrico y reducen el incremento radial anual. Este comportamiento fisiológico es común para especies arbóreas en las zonas semiáridas del norte de México y Suroeste de los estados Unidos de América, donde la precipitación constituye el factor más limitante para el crecimiento de la especie (Fritts, 1976).

Aunque T. mucronatum muestra cierta tolerancia a la sequía, la disponibilidad de agua entre los meses de marzo y septiembre, resulta esencial para el crecimiento de T. mucronatum, donde se presenta $75 \%$ de la lluvia anual (Mosiño \& García, 1974). La función de respuesta determinada para $T$. mucronatum en este sitio es relevante para su conservación, ya que un caudal ecológico estable en este período, que provea un volumen de agua que satisfaga el requerimiento hídrico de la especie, propiciará un adecuado desarrollo de T. mucronatum y permitirá el desarrollo de los procesos ecológi$\cos$, que conllevan a una adecuada provisión de servicios ecosistémicos (calidad de agua, recarga de mantos acuíferos, biodiversidad, captura de carbono, belleza escénica).

Reconstrucciones de precipitación con $T$. mucronatum son escasas en México y de temperatura son aún más limitadas. En este estudio, la reconstrucción de precipitación resalta 21 eventos secos y 22 húmedos. Eventos extremos de sequía ocurrieron en 1813-1818, 1863, 1870-1871, 1953, 1956 y 1974, sequías que coinciden con reconstrucciones generadas en el sur de Coahuila y en otras regiones del centro y norte de México (Stahle et al., 2016); mientras que los períodos húmedos ocurridos de 1828-1829, 1851, 1946, 1968, 1970-1972, 1979, 1981 y 1987, también se registran en una reconstrucción realizada para la Sierra de Zapalinamé, Coahuila (Constante, Villanueva, Cerano, Cornejo, \& Valencia, 2009). Gran parte de estos eventos se han reportado para la Sierra Madre Oriental y Occidental, cuyas cuencas generan escurrimientos para los Estados ubicados en el Golfo de México y Planicie Costera del Pacífico (Díaz, Therrell, Stahle,
\& Cleveland, 2002; Cleaveland, Stahle, Therrell, Villanueva-Díaz, \& Burns, 2003), lo que implica que este comportamiento, se deriva de tele-conexiones de fenómenos de circulación general como ENSO, cuyo efecto se deja sentir en grandes áreas y de manera simultánea en diversas regiones de México (Méndez \& Magaña, 2010).

La reconstrucción de temperatura máxima desarrollada permitió identificar periodos como el de 1950-1958, donde la temperatura superó a la media histórica reconstruida $\left(37.39^{\circ} \mathrm{C}\right)$, que, aunado a escasas precipitaciones, redujo la producción agrícola y favoreció la migración del campo a la ciudad y hacia los Estados Unidos de América (Florescano, 1980).

Las frecuencias a 2.7 y 45.9 años identificadas por el análisis de densidad espectral y el análisis de ondeleta, se encuentran en el dominio del fenómeno ENSO ( $\mathrm{Li}$ et al., 2011; Bruun, Allen, \& Smyth, 2017), donde en dichas frecuencias se han presentado los eventos de sequía más intensos en el norte de México (Cerano et al., 2011). Esta influencia no fue tan marcada en el noroeste de México, donde la correlación con el SOI reconstruido noviembre-febrero, aunque significativa $(\mathrm{r}=$ $-0.41, \mathrm{P}<0.01$ ), no se compara a la obtenida para zonas de la Sierra Madre Occidental en los estados de Chihuahua y Durango, que son cercanas a 0.7 (Díaz-Ramírez, Villanueva-Díaz, \& Cerano-Paredes, 2016). La precipitación invierno primavera en el noreste de México tiene más influencia del ingreso de frentes fríos y la presencia temprana de huracanes (Magaña et al., 1999).

La asociación entre la serie dendrocronológica de T. mucronatum y el índice de sequía SPEI fue significativa para el mes de junio, lo que permitió corroborar que el comportamiento de los componentes del SPEI (precipitación y evapotranspiración), afectan el incremento radial de la especie en la estación de verano; es decir, que las condiciones cálidas conducen a un crecimiento reducido de la especie, mientras que las condiciones frías mejoran la producción de biomasa (Jiang, Liu, Wu, \& Wang, 2016). La correlación espacial entre el índice SPEI 
y el ancho de anillo (Fig. 7), concuerda con lo reportado por Vicente-Serrano, Camarero, \& Azorin-Molina (2014), lo que confirma el hecho de que existe una amplia señal espacial entre los crecimientos de especies arbóreas, particularmente coníferas y las condiciones de sequía que prevalecen en la porción noreste de México y sureste de Estados Unidos. Sin embargo, la relación entre PDSI reconstruido y el crecimiento de la especie en estudio, mostró una relación positiva y significativa, así como una influencia espacial a gran escala, corroborando lo encontrado por el índice SPEI al presentarse un comportamiento regional similar.

La influencia del PDO y de AMO en el incremento radial de T. mucronatum no fue significativa, contrario a lo encontrado para otras cuencas del norte de México (Martínez-Sifuentes, Villanueva-Díaz, \& Estrada-Ávalos, 2020).

La generación de una serie dendrocronológica de T. mucronatum que se extiende por más de 200 años en la cuenca del Río Sabinas, Coahuila, permitió determinar las fluctuaciones en la variabilidad de la precipitación estacional abril-junio, donde se presenta el $34 \%$ de la precipitación anual, pero que es de gran relevancia para el crecimiento de la especie y explica más del $50 \%$ de la variabilidad en el incremento radial anual.

La temperatura máxima promedio juniojulio tuvo un efecto negativo en el crecimiento de la especie, al propiciar incrementos en la demanda evapotranspirativa y abatir el agua almacenada en el perfil del suelo en dicho período; este efecto se revierte cuando las temperaturas caen por debajo de $37.4{ }^{\circ} \mathrm{C}$, en cuyo caso favorecen el incremento radial de la especie, siempre y cuando exista humedad disponible para el proceso evapotranspirativo.

La reconstrucción de precipitación indicó la presencia de eventos secos y húmedos registrados en diversas reconstrucciones de lluvia desarrolladas para el noreste de México, lo que implica que la presencia de eventos extremos está gobernada por fenómenos climáticos de amplio alcance como es ENSO y en menor grado PDO y AMO.
La cronología de anillo total fue sensible a los índices de sequía SPEI de junio y PDSI reconstruido noviembre-febrero y PDSI calibrado, lo que indica la alta relación entre precipitación, disponibilidad y demanda hídrica.

La información generada sustenta propuestas técnicas para el desarrollo de planes enfocados a la conservación y restauración de bosques de galería de T. mucronatum en esta región, afectados por cambios drásticos en el uso del suelo, particularmente la liberación de un gasto ecológico para la sustentabilidad de la especie.

Declaración de ética: los autores declaran que todos están de acuerdo con esta publicación y que han hecho aportes que justifican su autoría; que no hay conflicto de interés de ningún tipo; y que han cumplido con todos los requisitos y procedimientos éticos y legales pertinentes. Todas las fuentes de financiamiento se detallan plena y claramente en la sección de agradecimientos. El respectivo documento legal firmado se encuentra en los archivos de la revista.

\section{AGRADECIMIENTOS}

Para el desarrollo de este estudio, se recibió financiamiento del CONACYT (FOINS), Problemas Nacionales, proyecto PN2016/2976, SEP-CONACYT/283134 y del proyecto CONACYT-SENER S0019 -2014-1/245410.

\section{RESUMEN}

Introducción: La determinación del clima en cuencas productoras de agua, como la del río Sabinas, Coahuila, donde no existe información de su variabilidad, se puede estimar mediante anillos de crecimiento de ahuehuete (Taxodium mucronatum Ten.), especie longeva con anillos bien definidos, presente en las zonas ribereñas, constituye un "proxy" o método indirecto de la variabilidad climática interanual y multianual. Objetivo: Desarrollar una reconstrucción estacional de precipitación y temperatura máxima y analizar la influencia que ejercen fenómenos de circulación global en el crecimiento de la especie. Métodos: Los núcleos de crecimiento de T. mucronatum fueron datados a través de técnicas dendrocronológicas para producir una serie de ancho de anillo. Se utilizaron datos de mallas del 
clima de dos bases de datos para desarrollar el análisis de la función de respuesta con fines de reconstrucción climática. Resultados: Una cronología de anillo total de 218 años (1808-2018) se desarrolló con especímenes de T. тисronatum en parajes del Río Sabinas, con la que se generó una reconstrucción estacional de precipitación abril-junio y de temperatura máxima junio-julio. El periodo detectado más seco se presentó de 1815 a 1818 con una precipitación de $27.39 \mathrm{~mm}$ y una temperatura máxima de $41.16{ }^{\circ} \mathrm{C}$; mientras que el año más húmedo fue 1828 con 393.72 $\mathrm{mm}$. La cronología de anillo total se correlacionó con los índices de sequía SPEI y PDSI, y con índices de fenómenos atmosféricos como El Niño Oscilación del Sur, a través del Índice de Oscilación del Sur (SOI) y el índice Multivariado (MEI); Oscilación Decadal del Pacífico (PDO) y Oscilación Multidecadal del Atlántico (AMO). La relación entre el índice de anillo total y el del Índice Estandarizado de Precipitación Evaporación (SPEI) mostró significancia en el mes de junio $(\mathrm{r}=0.52, \mathrm{P}<0.01)$, al igual que el Índice de Severidad de Sequía del Palmer (PDSI), PDSI anual ( $\mathrm{r}=$ $0.38, \mathrm{P}<0.05)$. El SOI reconstruido noviembre-febrero, se asoció significativamente con la serie dendrocronológica ( $\mathrm{r}$ $=-0.41, \mathrm{P}<0.01)$. La Oscilación Decadal del Pacífico y la Oscilación Multidecadal del Atlántico, no mostraron significancia. Conclusiones: En este estudio, desarrollamos una función de respuesta climática y reconstruimos variables climáticas estacionales (precipitación, temperatura máxima) de importancia para desarrollar estrategias de manejo para la conservación de T. mucronatum en esta cuenca, e implementar acciones de mitigación para la presencia de eventos climáticos extremos que se pueden presentar en los próximos años.

Palabras clave: Ahuehuete; anillos de árboles; reconstrucción climática; eventos extremos.

\section{REFERENCIAS}

Anderson, S.R., Ogle, I.R., Tootle, G., \& Oubeidillah, A. (2019). Tree-Ring Reconstructions of Streamflow for the Tennessee Valley. Hydrology, 6(2), 34. DOI: 10.3390/hydrology6020034.

Biondi, F., \& Waikul, K. (2004). DENDROCLIM2002: A $\mathrm{C}++$ program for statistical calibration of climate signals in tree-ring chronologies. Computers \& Geosciences, 30, 303-311. DOI: 10.1016/j. cageo.2003.11.004

Bruun, J.T., Allen, J.I., \& Smyth, T.J. (2017). Heartbeat of the Southern Oscillation explains ENSO climate resonances. Journal of Geophysics Research: Oceans, 122, 6746-6772. DOI: 10.1002/2017JC012892

Bunn, A., Korpela, M., Biondi, F., Campelo, F., Mérian, P., Qeadan, F., \& Schulz, M. (2019). Dendrochronology Program Library in R. Retrieved from https://cran.rproject.org/web/packages/dplR/vignettes/intro-dplR. pdf
Castruita-Esparza, L.U., Silva, L.C.R., Gómez-Guerrero, A., Villanueva-Diaz, J., Correa-Diaz, A., \& Horwath, W.R. (2019). Coping with extreme events: growth and wáter-use efficiency of trees in western Mexico during the driest and wettest periods of the past one hundred sixty years. Journal of Geophysical Research: Biogeosciences, 124, 3419-3431. DOI: 10.1029/2019JG005294

Cerano, J., Villanueva, J., Valdez, R.D., Cornejo, E.H., Sánchez, I., \& Constante, V. (2011). Variabilidad histórica de la precipitación reconstruida con anillos de árboles para el sureste de Coahuila. Revista Mexicana de Ciencias Forestales, 2(4), 33-47. DOI: 10.29298/ rmcf.v2i4.599

Cleaveland, M.K., Stahle, D.W., Therrell, M.D., Villanueva-Díaz, J., \& Burns, B.T. (2003). Tree-Ring Reconstructed Winter Precipitation and Tropical Teleconnections in Durango, Mexico. Climate Change, 59(3), 369-388. DOI: 10.1023/A:1024835630188

Comisión Nacional del Agua. (2013). Estadísticas agrícolas de los distritos de riego: año agrícola 2011-2012. México, D.F., México: Secretaría del Medio Ambiente y Recursos Naturales. Retrieved from http:// www.conagua.gob.mx/CONAGUA07/Publicaciones/ Publicaciones/SGIH-4-13.pdf

Constante, V., Villanueva, J., Cerano, J., Cornejo, E.H., \& Valencia, S. (2009). Dendrocronología de Pinus cembroides Zucc. y reconstrucción de precipitación estacional para el sureste de Coahuila. Revista Ciencia Forestal en México, 34(106), 17-39.

Cook, E.R. (1987). The decomposition of tree-ring series for environmental studies. Tree Ring Bulletin, 47, 37-59. Retrieved from http://hdl/handle. net/1050/261788

Correa-Díaz, A., Cómez-Guerrero, A., Villanueva-Díaz, J., Castruita-Esparza, L.U., Martínez-Trinidad, T., \& Cervantes-Martínez, R. (2014). Análisis dendroclimático de ahuehuete (Taxodium mucronatum Ten.) en el centro de México. Agrociencia, 48, 537-551.

Díaz, S.C., Therrell, M.D., Stahle, D.W., \& Cleaveland, M.K. (2002). Chihuahua (Mexico) winter-spring precipitation reconstructed from tree-rings, 16471992. Climate Research, 22, 237-244. DOI: 10.3354/ cr022237

Díaz-Ramírez, B., Villanueva-Díaz, J., \& Cerano-Paredes, J. (2016). Reconstrucción de la precipitación estacional con anillos de crecimiento para la región hidrológica Presidio-San Pedro. Madera y Bosques, 22, 111-123.

Florescano, E.M. (1980). Análisis histórico de las sequías en México. México D.F., México: Secretaría de Agricultura y Recursos Hidráulicos.

Fritts, H.C. (1976). Tree-rings and climate. New York, United States: Academic Press. 
García, E. (2004). Modificaciones al sistema de clasificación climática de Köppen (5ta ed.). México D.F., México: Instituto de Geografía, Universidad Nacional Autónoma de México.

Granados-Sánchez, D., Hernández-García, M.A., \& LópezRíos, G.F. (2006). Ecología de las zonas ribereñas. Revista Chapingo Serie Ciencias Forestales y del Ambiente, 12(1), 55-69.

Grinsted, A., Moore, J.C., \& Jevrejeva, S. (2004). Application of the cross wavelet transform and wavelet coherence to geophysical time series. Nonlinear Processes in Geophysics, European Geosciences Union (EGU), 11, 561-566. Retrieved from https:// hal.archives-ouvertes.fr/hal-00302394

Harris, I., Jones, P.D., Osborn, T.J., \& Lister, D.H. (2014). Updated high-resolution grids of monthly climatic observations-the CRU TS3.10 Dataset. International Journal of Climatology, 34(3), 623-642. DOI: 10.1002/joc. 3711

Holmes, R.L. (1983). Computer-assisted quality control in tree-ring dating and measurement. Tree Ring Bulletin, $43,69-78$.

INEGI. (1983). Sintesis geográfica del Estado de Coahuila. México D.F., México: Dirección General de Geografía.

Jiang, P., Liu, H., Wu, X., \& Wang, H. (2016). Tree-ringbased SPEI reconstruction in central Tianshan Mountains of China since AD 1820 and links to westerly circulation. Journal of Climatology, 37, 2863-2872. DOI: $10.1002 /$ joc. 4884 .

Li, J., Xie, S.P., Cook, E.R., Huang, G., D’Arrigo, R., Liu, F., \& Zheng, X.T. (2011). Interdecadal modulatrion of El Niño amplitude during the past millennium. Nature Climate Change, 1, 114-118. DOI: 10.1038/ nclimate 1086.

Magaña, V., Pérez, J.L., Vázquez, L.L., Carrizosa, E., \& Pérez, J. (1999). El Niño y el clima. In V. Magaña (Ed.), Los impactos del Niño en México (pp. 229). México D.F., México: SEP-CONACYT.

Mantua, N.J., Hare, S.R., Zhang, Y., Wallace, J.M., \& Francis, R.C. (1997). A pacific interdecadal climate oscillation with impacts on salmon production. Bulletin of American Meteorology Society, 78, 1069-1080. DOI: $10.1175 / 1520-0477(1997) 078 \% 3 C 1069$ :APIC OW\%3E2.0.CO;2

Martínez, M. (1963). Las pinaceas mexicanas ( $3^{\mathrm{a}}$ Ed.). México, D.F., México: Instituto de Biología, Universidad Autónoma de México.

Martínez-Sifuentes, A.R., Villanueva-Díaz, J., \& EstradaÁvalos, J. (2020). Runoff reconstruction and climatic influence with tree rings, in the Mayo river basin, Sonora, Mexico. iForest, 13, 98-106. DOI: 10.3832/ ifor3190-013
Méndez, M., \& Magaña, V. (2010). Regional aspects of prolonged meteorological droughts over Mexico and Central America. Journal of Climate, 23, 1175-1188. DOI: 10.1175/2009/CL13080.1

Mérian, P., Pierrat, J.C., \& Lebourgeois, F. (2013). Effect of sampling effort on the regional chronology statistics and climate-growth relationships estimation. Dendrochronologia, 31(1), 58-67. DOI: 10.1016/j. dendero.2012.07.001

Mosiño, P., \& García, E. (1974). The climate of Mexico. Climates of North America. World Survey of Climatology, 11, 345-404.

Osorio-Osorio, J.A., Astudillo-Sánchez, C.C., VillanuevaDíaz, J., Soria-Díaz, L., \& Vargas-Tristan, L.V. (2020). Reconstrucción histórica de la precipitación en la Reserva de la Biosfera El Cielo, México, mediante anillos de crecimiento en Taxodium mисronatum (Cupressaceae). Revista de Biología Tropical, 68(3), 818-832.

R Core Team. (2014). $R$ : A language and environment for statistical computing. $R$ Foundation for Statistical Computing, Vienna, Austria. Retrieved from http:// www.R-project.org/

Ropelewski, C.F., \& Jones, P.D. (1987). An extension of the Tahiti-Darwin Southern oscillation Index. Monthly Weather Review, 115, 2161-2165. DOI: $10.1175 / 1520.0493(1987)<2161$ :AEOTTS $>2.0$ CO:2

Seager, R., Ting, M., Davis, M., Cane, M., Nike, M., Nakumara, J., \& Stahle, D.W. (2009). Mexican drought: an observational modeling and tree ring study of variability and climate change. Atmósfera, 22(1), 1-31.

Serrano-Barrios, L., Vicente-Serrano, S.M., Flores-Magdaleno, H., Tijerina-Chávez, L., \& Vázquez-Soto, D. (2016). Variabilidad espacio-temporal de las sequías en la cuenca pacífico norte de México (1961-2010). Cuadernos de Investigación Geográfica, 42(1), 185 204. DOI: $10.18172 /$ cig. 2857

Stahle, D.W., Villanueva-Díaz, J., Burnette, D.J., CeranoParedes, J., Heim, R.R. Jr., Fye F.K., \& Stahle, D.K. (2011). Major Mesoamerican droughts of the past millennium. Geophysical Research Letters, 38, L05703. DOI: 10.1029/2010GL046472

Stahle, D., Burnette, D.J, Villanueva-Díaz, J., Cerano-Paredes, J., Fye, F.K., Griffin, R.D. \& Wolff, K. (2012). Tree-ring analysis of ancient baldcypress trees and subfossil wood. Quaternary Science Reviews, 34, 1-15. DOI: 10.1016/j.quascirev.2011.11.005

Stahle, D.W., Cook, E.R., Burnette, D.J., VillanuevaDiaz, J., Cerano-Paredes, J., Burns, J.N., \& Howard, I.M. (2016). The Mexican Drought Atlas: treering reconstructions of the soil moisture balance during the late preHispanic, colonial, and modern eras. Quaternary Science Review, 149, 34-60. DOI: jquascirev.2016.06.018 
Stahle, D.W., Edmondson, J.R., Howard, I.M., Robbins, C.R., Griffin, R.D., Carl, A., \& Torberson, M.C.A (2019). Longevity, climate sensitivity, and conservation status of wetland trees at Black River, North Carolina. Environmental Research Communications, 1, 041002. DOI: 10.1088/2515-7620/abOc4a

Stokes, M.A., \& Smiley, T.L. (1968). An Introduction to Tree-Ring Dating. Chicago, United States: University of Chicago Press.

Tarik, C.G., Aslak, G., \& Viliam, S. (2017). R package "biwavelet": conduct univariate and bivariate wavelet analyses (Version 0.20.15). Retrieved from https:// github.com/tgouhier/biwavelet.

Terán-Cuevas, A. (2010). Escenarios de lluvia en México (Tesis de doctorado). Centro Interdisciplinario de Investigaciones y Estudios sobre Medio Ambiente y Desarrollo (CIIEMAD), México D.F., México.

Trenberth, K., \& Zhang, R. (2019). The Climate Data Guide: Atlantic Multi-decadal Oscillation (AMO). Retrieved from https://climatedataguide.ucar.edu/ climate-data/atlantic-multi-decadal-oscillation-amo

Van Der Schrier, G., Barichivich, J., Briffa, K.R., \& Jones, P.D. (2013). A scPDSI-bases global data set of dry and wet spells for 1901-2009. Journal Geophysical Research: Atmosphere, 118, 4025-4048. DOI: 10.1002/jgrd.50355.

Vicente-Serrano, S.M., Beguería, S., López-Moreno, J.I., Angulo, M., \& Kenawy, A.E. (2010). A new global $0.5^{\circ}$ gridded dataset (1901-2006) of a multiscalar drought index: comparison with current drought index datasets based on the palmer drought severity index. Journal of Hydrometeorology, 11, 1033-1043.

Vicente-Serrano, S., Camarero, J., \& Azorin-Molina, C. (2014). Diverse responses of forest growth to drought time-scales in the Northern Hemisphere. Global Ecology and Biogeography, 23, 1019-1030. DOI: 10.1111 /geb. 12183

Villanueva-Díaz, J., Stahle, D.W., Luckman, B.H., Cerano, J., Therrell, M.D, Morán, R., \& Cleaveland, M.K. (2007). Potencial dendrocronológico de Taxodium mucronatum Ten. y acciones para su conservación en México. Ciencia Forestal, 32(101), 9-37.
Villanueva-Díaz, J., Constante, V., Cerano, J., Estrada, J., \& Tostado, M. (2013). Impacto de las represas en Taxodium mucronatum T. en el río San Pedro-Mezquital, Durango. Revista Mexicana de Ciencias Forestales, 4(18), 44-56. DOI: 10.29298/rmcfv4i18.388

Villanueva-Díaz, J., Constante, V., Cerano, J., \& Martínez, A. (2014). La cuenca San Martín y situación del ahuehuete (Taxodium mucronatum Ten.) en el río Sabinas, Coahuila. Coyoacán, México: Instituto Nacional de Investigaciones Forestales Agrícolas y Pecuarias.

Villanueva-Díaz, J., Stahle, D.W., Therrell, M.D., Beramendi-Orosco, L., Estrada-Avalos, J., MartinezSifuentes, A.R., ... Cerano-Paredes, J. (2020). The climatic response of baldcypress (Taxodium mucronatum Ten.) in San Luis Potosi, Mexico. Trees, 34, 623-635. DOI: 10.1007/s00468-019-01944-0.

Villareal, J.A., Carranza, M.A., Estrada, E.C., \& Rodríguez, A. (2006). Flora riparia de los ríos Sabinas y San Rodrigo, Coahuila, México. Acta Botánica Mexicana, 75, 1-20. DOI: 10.21829/abm75.2006.1012

Wang, T., Hamann, A., Spittlehouse, D.L., \& Carroll, C. (2016). Locally downscaled and spatially customizable climate data for historical and future periods for North America. PLoS One, 11, e0156720. DOI: 10.1371/journal.pone. 0156720

Wells, N., Goddard, S., \& Hayes, M.J. (2004) A selfcalibrating Palmer Drought Severity Index. Journal of Climate, 17, 2335-2351. DOI: 10.1175/1520-0442(2004)017<2335:ASPDSI $>2.0$. $\mathrm{CO} ; 2$.

Wolter, K., \& Timlin, M.S. (2011). El Niño/Southern Oscillation behavior since 1871 as diagnosed in an extended multivariate ENSO index (MEI.ext). International Journal of Climatology, 31, 1074-1087. DOI: $10.1002 /$ joc. 2336 .

Woodhouse, C., \& Lukas, J. (2006). Drought, tree rings and water resource management in Colorado. Canadian Water Resources Journal, 31, 297-310. DOI: 10.4296/cwrj3104297 